\title{
Complex Renal Arteriovenous Malformation (AVM) Embolization using Ethylene Vinyl Alcohol (EVOH) Copolymer and a Balloon Microcatheter
}

\author{
Heather Kate Moriarty ${ }^{1}$ Warren Clements ${ }^{1,2}$ \\ ${ }^{1}$ Department of Radiology, Alfred hospital, Melbourne, Australia \\ ${ }^{2}$ Department of Surgery, Monash University, Clayton, Australia
}

J Clin Interv Radiol ISVIR 2019;3:134-138

\begin{abstract}
Address for correspondence Heather Kate Moriarty, MB, BCh, BAO, MMedSc, MRCPI, FFRRCSI, Interventional Radiology Fellow, The Alfred Hospital, Department of Radiology, 55 Commercial Road, Melbourne, VIC 3004, Australia (e-mail: heather.moriarty@gmail.com).
\end{abstract}

\author{
Abstract \\ Keywords \\ - renal intervention \\ - arteriovenous \\ malformation \\ - microballoon catheter \\ Scepter XC
}

Occurrence of a symptomatic renal arteriovenous malformation (AVM) is rare. The authors present the case of a patient with a background of Turner's syndrome, hemophilia B, and horseshoe kidney, who presented with hematuria and was successfully treated with endovascular embolization. The use of a microballoon catheter Scepter XC (Microvention, Inc.) to safely embolize the feeding arterial supply, using ethylene vinyl alcohol (EVOH) copolymer (Onyx; Medtronic/ev3) has not, to the authors' knowledge, previously been described for the treatment of renal AVM. This novel use of an occlusal balloon catheter is permitted by the precipitation rate of Onyx, and allowed a safe and effective procedure, reducing the risk of reflux, allowing a forward push of embolic material in a controlled manner.

\section{Introduction}

Turner's syndrome is a chromosome anomaly with a prevalence of $1: 2,500$ female children. It is reported that 45 to $50 \%$ of patients with Turner's syndrome have cardiovascular anomalies. Horseshoe kidney occurs in approximately $21.2 \%$ of people with Turner's syndrome. ${ }^{1}$

Renal arteriovenous malformations (AVMs) are abnormal communications between the intrarenal arterial and venous systems. The incidence ranges from 1 per 1,000 patients to 1 per 2,500. Classification may be based on angiographic appearance of the AVM, type IIIb AVM, have multiple shunts between the arterioles and venules, forming a complex vascular network, with a dilated fistulous channels. These appearances can also be classified as a congenital cirsoid AVM. ${ }^{2}$ Treatment options include conservative management, which may be appropriate in asymptomatic patients with small AVMs. Traditional management of symptomatic AVMs has been surgical; however, endovascular management has been successful and is increasingly the treatment of choice in the occlusion of renal and visceral AVMs.
We present a case of a 22-year-old female who underwent embolization of a cirsoid renal arteriovenous malformation using ethylene vinyl alcohol (EVOH) copolymer (Onyx; Medtronic/ev3), delivered via an occlusal, dual lumen balloon microcatheter Scepter XC (Microvention, Inc.)

Onyx is an uncommon but emerging treatment modality for visceral vascular malformations, especially in cases in which delicate nidal penetration of the AVM is required., The use of an occlusal balloon catheter is permitted by the precipitation rate of Onyx, and in turn permits the safe and complete embolization of the AVM, using sufficient forward pressure without risk of reflux.

\section{Case Report}

Informed consent was obtained from the patient. A 22-yearold female presented with macroscopic hematuria, ongoing for 8 days. On presentation to the Emergency Department, the patient's blood pressure was recorded at 118/49 and there was a tachycardia of 104 beats per minute. Her hemoglobin was $82 \mathrm{~g} / \mathrm{L}$ on presentation. Her medical history included Turner's syndrome, horseshoe kidney, cirrhosis with portal
License terms

$10.1055 / \mathrm{s}-0039-1694094$

accepted after revision

ISSN 2457-0214. 
hypertension, splenomegaly, and prior endovascular embolization of a hepatic vascular abnormality. The patient was managed initially with fluid resuscitation including blood transfusion and factor replacement.

A computed tomography (CT) angiogram ( - Fig. 1) demonstrated the known horseshoe kidney and a vascular lesion in the lower pole of the kidney, to the left of midline with features suggestive of an AVM. Diagnostic renal angiography con-

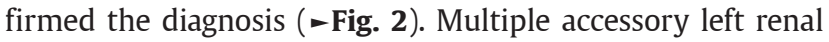
arteries were demonstrated. The AVM was supplied from numerous small branch arteries arising from the lowermost accessory renal left artery. The venous drainage was via multiple venous channels. Appearances were consistent with a Type $3 \mathrm{~b}$ or cirsoid type AVM. Ectasia of the hepatic and splenic arteries, and a splenic artery aneurysm were also visualized.

Subsequent to discussion at a multi-disciplinary team meeting, an endovascular approach was favored to treat the complex AVM.

Venous access was obtained via the right internal jugular vein, to establish the extent and number of venous outflow channels. Embolization by occlusion of the venous outflow, if feasible, could have been attempted. However, given the numerous venous outflow channels, the decision was made to approach the lesion from the arterial inflow. A catheter and sheath were advanced to the left renal vein. Venogram
(-Fig. 3) demonstrated early venous drainage from the AVM was via multiple small venous channels draining to the left renal vein and to a larger draining vein connected to the left common iliac vein. Arterial access was obtained via the right femoral artery and 6 French sheath inserted. A 5 French SIM 2 catheter (Impress Merit Medical) was placed in the lowermost accessory left renal artery. A dimethyl sulfoxide (DMSO) compatible Scepter XC 4 x $11 \mathrm{~mm}$ balloon microcatheter was advanced over a Synchro ${ }^{4}$ Soft microwire (Stryker Neurovascular) into the proximal accessory left renal artery and angiography performed. Subsequently, the balloon was inflated in the proximal vessel. After the catheter dead space was filled with DMSO, Onyx was slowly injected under fluoroscopic guidance ( - Fig. 4 ) with gentle forward pressure. A total of $2.5 \mathrm{~mL}$ of Onyx 18 was administered to satisfactorily occlude the AVM. The occlusion balloon was deflated and angiography performed in the treated accessory renal artery and in the aorta, demonstrating no residual vascular malformation (-Fig. 5).

Angioembolization was well tolerated by the patient. There was small volume of normal renal parenchyma infarcted, estimated to be less than $5 \%$ of the total renal volume. Within 7 days, macrohematuria ceased and the patient was discharged 10 days post procedure. At 6-month clinical follow-up, she remains asymptomatic without recurrence of hematuria.

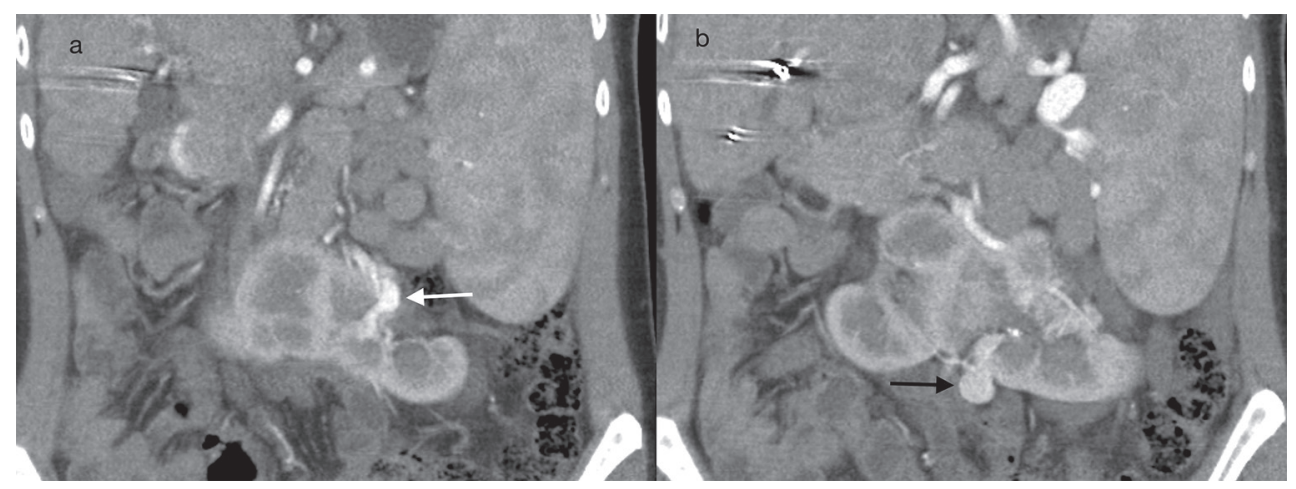

Fig. 1 Coronal computed tomography depicting the horseshoe kidney with AVM in the inferior aspect of the kidney (a, b). Arterial inflow (white arrow) and early draining veins (black arrow) are demonstrated.

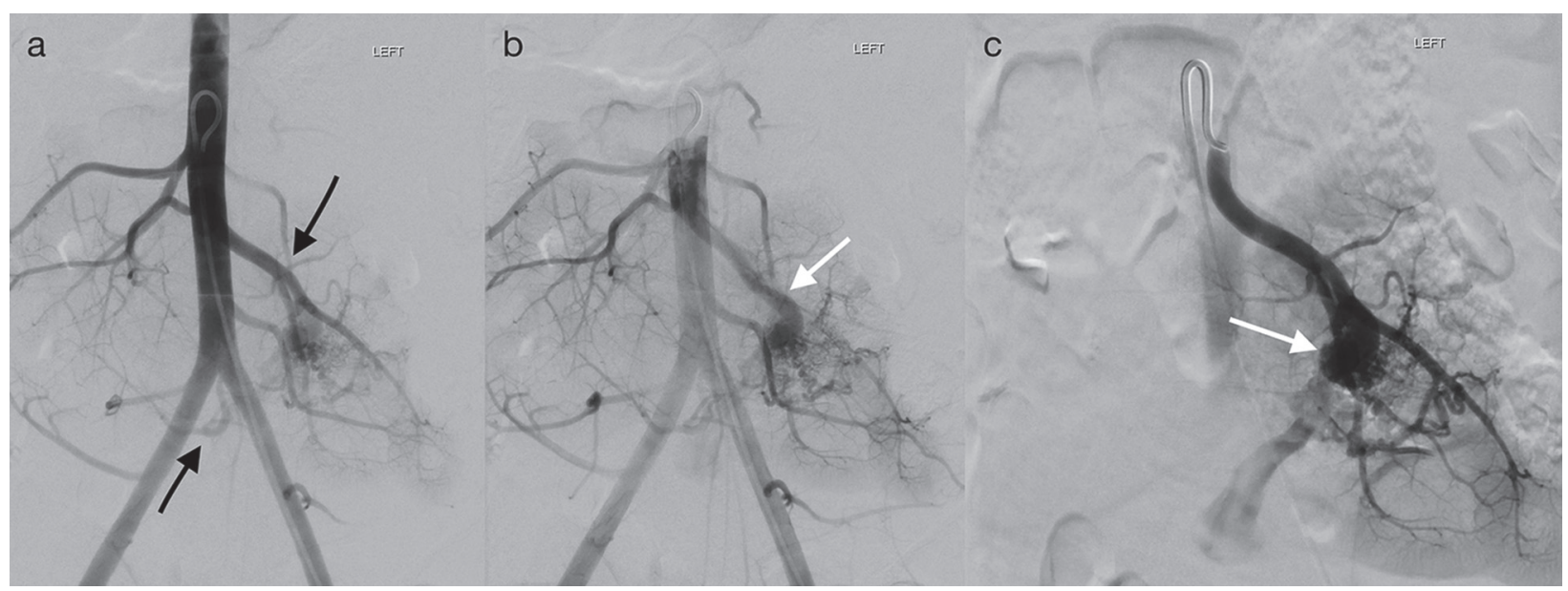

Fig. 2 Angiography demonstrating the arterial supply (a) to the renal AVM via an accessory lower pole renal artery and the median sacral artery (black arrows) and (b, c) early venous drainage (white arrows). 


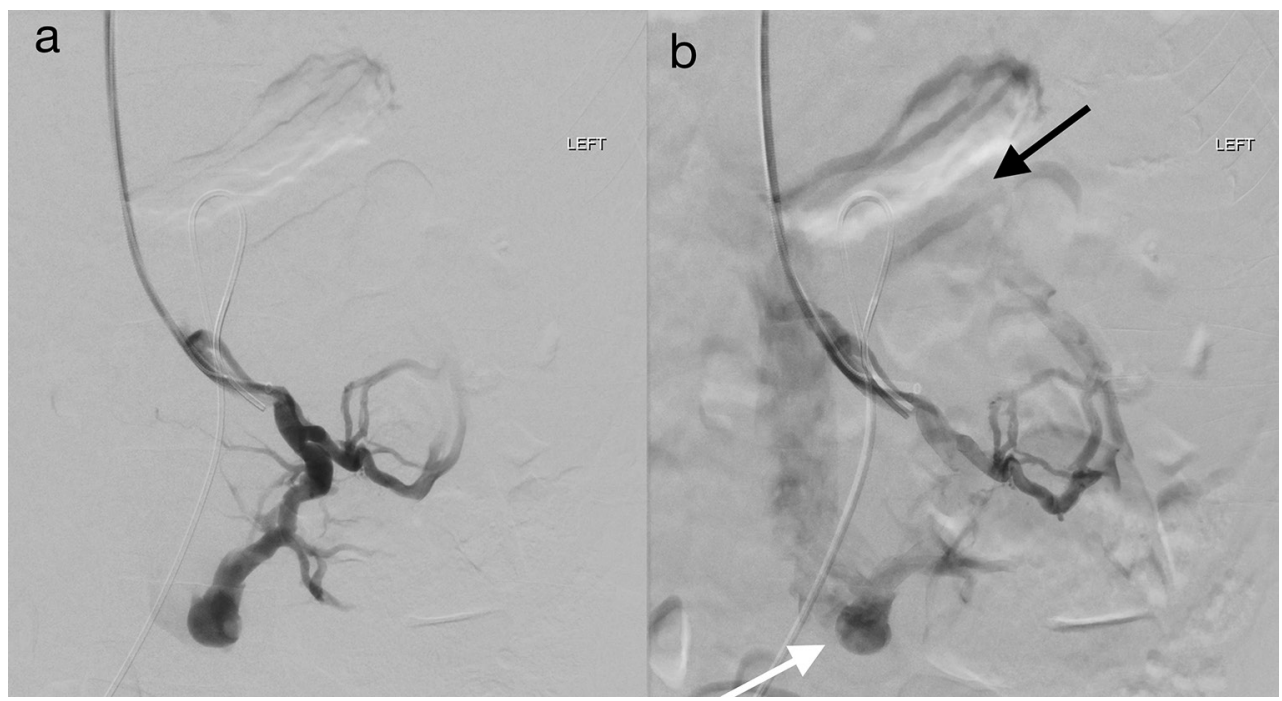

Fig. 3 (a, b) Venogram via catheter placed in a draining vein demonstrates venous drainage is via multiple dilated venous channels, draining to the left renal vein (white arrow) and the left common iliac vein (black arrow).

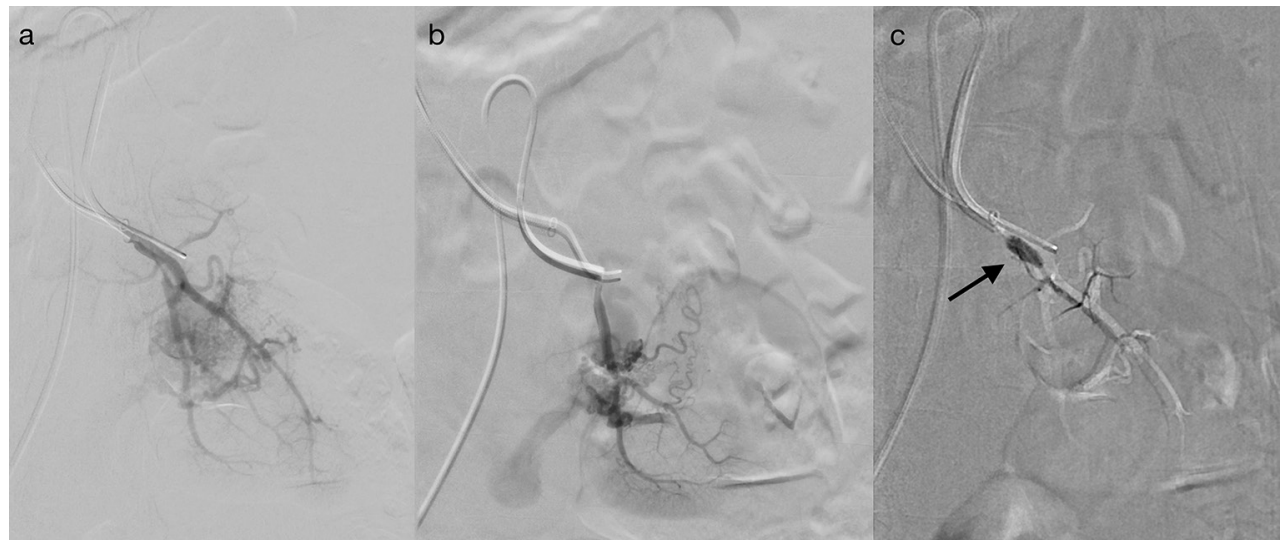

Fig. 4 Angiography in the main arterial inflow vessel depicting the arterial supply with typical cirsoid features (a) and early venous drainage (b). Embolization with Onyx (c), arrow depicts the position of the Scepter XC balloon microcatheter.

\section{Discussion}

There are various embolic agents available for the proceduralist to choose from when treating a patient with a visceral AVM. Frequently for the embolization of AVMs, as in this instance, coils or plugs were considered unsuitable as they would be unable to reach the nidus of the AVM. Particles would achieve greater nidal penetration than coils, however, there is a higher risk of nontarget embolization, due to particle embolization through the AVM and back through the systemic venous system. Opting for a largesized particle to avoid systemic embolization would simultaneously increase the risk of incomplete embolization because of incomplete nidal penetration. Liquid embolic agents are commonly used to treat renal AVMs, as they offer the benefit of quickly and effectively embolizing the nidus and often complex AVM vessels. Many liquid embolic agents have been described, such as, gelfoam, ethanol and n-butyl-cyano-acrylate (nBCA). ${ }^{5,6}$ The disadvantages of gelfoam includes potential recanalization of the vessel. nBCA is frequently used, and can reach the nidus of the AVM.
The nBCA can be mixed with lipiodol to produce varying consistency; however, in our experience, it can have unpredictable flow dynamics and thus difficult to control. In this situation, with a large and complex AVM, the risk of nBCA solidifying prior to reaching the nidus. Due to the swift precipitation of nBCA and ensuing risk of gluing the catheter in position, occlusion balloon catheters are not commonly used in for the delivery of nBCA. Alcohol and sclerosing agents are embolic agents, which would freely penetrate to the AVM nidus, however, there is a higher risk of complications from tissue necrosis. In this instance, there was a high risk of embolizing the arterial supply to a small volume of normal renal parenchyma, thus alcohol was considered unsuitable.

Onyx is a copolymer of EVOH. It has a longer precipitation rate, with more predictable flow, when compared to nBCA and alcohol. Thus it has both the ability to reach nidus in a controlled manner, and it is safe to use with an occlusal balloon microcatheter. Only DMSO compatible microcatheters should be used to deliver Onyx. It is a liquid embolic that has been predominantly utilized for the treatment of 


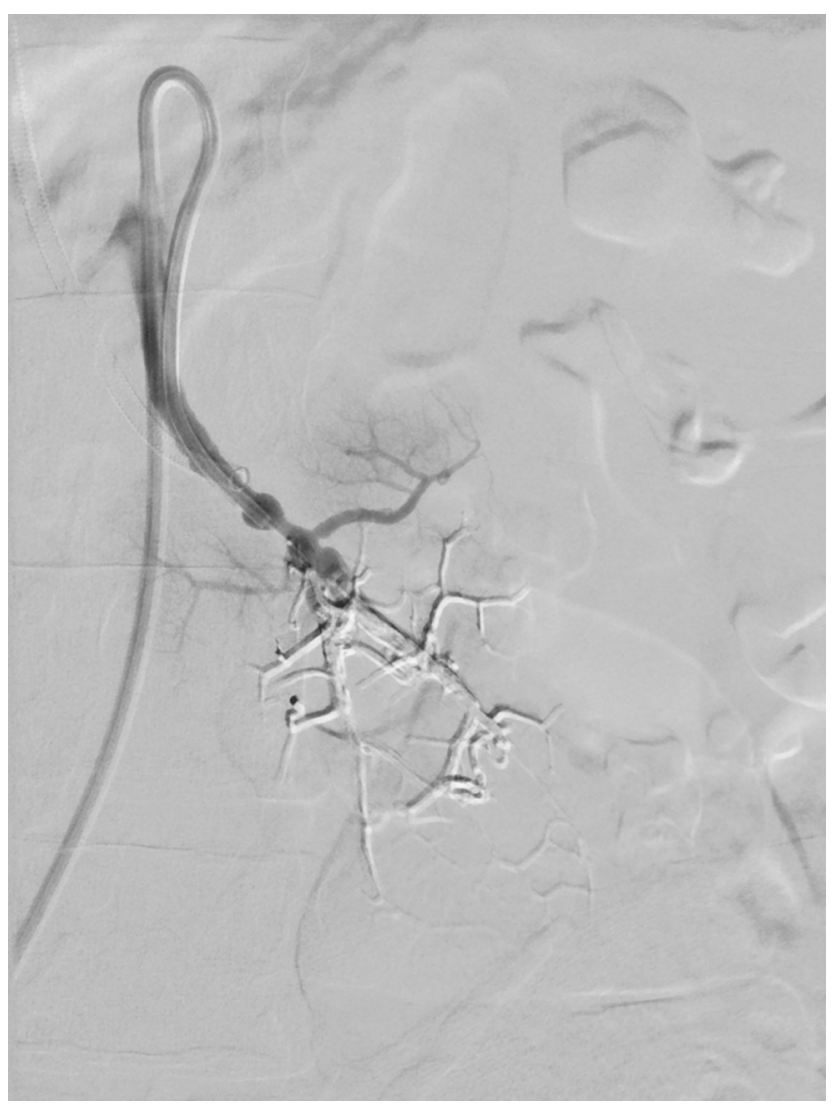

Fig. 5 Post embolization with Onyx, the Scepter XC balloon microcatheter has been removed and angiogram performed via the SIM 2. This demonstrates occlusion of the AVM with a cast evident in the embolized feeding arteries.

intracranial vascular abnormalities. ${ }^{7}$ There are a number of published cases using Onyx as the embolic agent for visceral vascular malformations, the treatment of acute hemorrhage, and also for embolization of renal AVMs. ${ }^{5}$ The use of an occlusal balloon can substantially decrease the risk of reflux of the embolic agent and allow an extensive cast within the renal AVM to be safely formed. ${ }^{8}$ Complications of balloon occlusion include injury to the vessel, such as dissection and rupture. The Scepter XC balloon microcatheter was chosen in this instance due to its compliance. Precautions to take while using occlusion balloon microcatheters include accurate measurement of vessel size to minimize the risk of vascular injury if oversized, or the risk of reflux due to inadequate seal with an under sized balloon. A test injection is recommended to ensure adequate occlusion. Considerations in the use of Onyx also however, include its relatively high cost, in comparison to other embolic agents and potential artefact on subsequent CT imaging.

\section{Conclusion}

This report depicts the safe and successful embolization of a Type $3 \mathrm{~b}$ renal AVM with Onyx using a balloon occlusal microcatheter. The flow of Onyx allowed effective embolization of the AVM nidus and feeding vessels, with a lower risk of embolus. The use of an occlusal balloon catheter is permitted by the precipitation rate of Onyx, and may allow a safe and effective procedure as it reduces or negates the risk of reflux, allowing a forward push of embolic material in a controlled manner.

\section{Conflicts of Interest}

On behalf of all authors, the corresponding author states that there is no conflict of interest.

\section{Declarations}

- The authors declare that there has been no duplicate publication or submission of any part of the work.

- The authors declare that all authors have read and approved the manuscript.

- The authors declare that there is no financial arrangement or other relationship that could be construed as a conflict of interest.

\section{Compliance with Ethical Standards}

- This study was not supported by any funding.

- Ethical approval: for this type of study, formal consent is not required. However, all procedures performed in studies involving human participants were in accordance with the ethical standards of the institutional and/or national research committee and with the 1964 Helsinki Declaration and its later amendments or comparable ethical standards. The study was performed in accordance with guidelines from the Alfred Hospital Research and Ethics Committee. This material is not under submission elsewhere.

- Informed consent was obtained from all individual participants included in the study.

- Consent for publication was obtained for every individual person's data included in the study.

\section{References}

1 Carvalho AB, Guerra Júnior G, Baptista MT, de Faria AP, Marini SH, Guerra AT. Cardiovascular and renal anomalies in Turner's syndrome. Rev Assoc Med Bras (1992) 2010;56(6):655-659

2 Cho SK, Do YS, Shin SW, et al. Arteriovenous malformations of the body and extremities: analysis of therapeutic outcomes and approaches according to a modified angiographic classification. J Endovasc Ther 2006;13(4):527-538

3 Juan YH, Lin YC, Sheng TW, et al. Application of onyx for renal arteriovenous malformation with first case report of a renal hyperdense striation sign: a CARE-compliant article. Medicine (Baltimore) 2015;94(39):e1658

4 Wetter A, Schlunz-Hendann M, Meila D, Rohde D, Brassel F. Endovascular treatment of a renal arteriovenous malformation with Onyx. Cardiovasc Intervent Radiol 2012;35(1):211-214

5 Maruno M, Kiyosue H, Tanoue S, et al. Renal arteriovenous shunts: clinical features, imaging appearance, and transcatheter embolization based on angioarchitecture. Radiographics 2016;36(2):580-595

6 Kimura Y, Osuga K, Ono Y, Nakazawa T, Higashihara H, Tomiyama N. Long-term outcomes of selective renal artery 
embolization for renal arteriovenous fistulae with dilated venous sac. J Vasc Interv Radiol 2018;29(7):952-957

7 McConnell KA, Tjoumakaris SI, Allen J, et al. Neuroendovascular management of dural arteriovenous malformations. Neurosurg Clin N Am 2009;20(4):431-439
8 Kang F, Shah K, Park H, et al. 3:18 PM Abstract No. 103 Balloon-assisted Onyx embolization of high-flow peripheral arteriovenous malformations with dual-lumen coaxial balloon microcatheter: technical aspects and clinical results. J Vasc Interv Radiol 2018;29(4):S48 